\title{
Relationship in sign between tilt and twist in active region magnetic fields
}

\author{
L. Tian, S. Bao, H. Zhang, and H. Wang \\ National Astronomical Observatories, Chinese Academy of Sciences, Beijing 100012, PR China \\ Received 15 September 2000 / Accepted 24 April 2001
}

\begin{abstract}
In this paper, we analyze the photospheric vector magnetograms of 286 active regions (ARs) which were observed from 1988-1996 during the 22nd solar cycle at Huairou Solar Observing Station in Beijing. Among these active regions, which are dominated by bipolar magnetic fields, 203 ARs exhibit a simple configuration of magnetic fields, while 83 ARs show a more complicated configuration of magnetic fields. When we explore the relationship between the sign of the tilt angle of magnetic polarity axis and the sign of the mean current helicity defined by $\left\langle\boldsymbol{B}_{\|} \cdot(\nabla \times \boldsymbol{B})_{\|}\right\rangle$, we find that: (1) There is a negative correlation between the sign of the tilt angle and the sign of the current helicity if the tilt angle is set a positive value $\left(0-90^{\circ}\right)$ in the northern hemisphere and a negative value $\left(0--90^{\circ}\right)$ in the southern hemisphere for active regions following Joy's law. In other words, about $60 \%$ of the bipolar active regions have a positive/negative tilt angle with respect to solar equator and a negative/positive current helicity in the northern/southern hemisphere. (2) In about one-third of the bipolar active regions, the tilt angle and the current helicity have the same sign in both hemispheres. They are abnormal active regions with an "abnormal chirality". These active regions are located preferentially in some longitudinal bins. (3) Most of the 62 X-ray flares larger than M-class during the 22nd solar cycle have a tendency to locate in some longitudinal bins, where active regions with "abnormal chirality" appear frequently.
\end{abstract}

Key words. Sun: magnetic field - Sun: photosphere - Sun: interior

\section{Introduction}

It is commonly believed that magnetic fields in the solar atmosphere are caused by a dynamo operating at or beneath the bottom of the convection zone and then emerge as $\Omega$-flux tubes on the solar surface to form solar active regions. Joy's Law (Hale et al. 1919) states that in many active regions of bipolar magnetic fields, the magnetic polarity axis has a particular inclination, with the preceding spot closer to the solar equator than the following spot. The angle between the magnetic polarity axis and the solar equator is defined as the "tilt angle". Observational studies of the tilt angle have been published in many papers (Howard 1989, 1991; Wang \& Sheeley 1989; Tian et al. 1999). The physical origin of the tilt angle has been discussed by following authors: Babcock (1961) proposed that, if the interior field has a purely toroidal orientation, Coriolis forces acting on the upwelling fluid associated with an active region would produce a tilt in the sense that is measured. Schmidt (1968) and Wang \& Sheeley (1991) suggested that the tilts of active regions are formed by the Coriolis force acting on the expanding plasma contained within a buoyant,

Send offprint requests to: L. R. Tian,

e-mail: tlr@ourstar.bao.ac.cn rising flux tube, rather than on the surrounding fields. On the other hand, the solar magnetic fields are twisted. The twist has been inferred by a number of methods including studying the morphology of filaments (Martin et al. 1994) and coronal loops (Rust \& Kumar 1996), in situ measurements of flux ropes (Burlaga 1988) and interplanetary fields (Bieber et al. 1987), computing the force-free parameter $\alpha_{\text {best }}$ (Pevtsov et al. 1995) and mean current helicity density $<h_{\|}>=<(\nabla \times \boldsymbol{B})_{\|} \cdot \boldsymbol{B}_{\|}>$(Abramenko et al. 1996; Bao \& Zhang 1998). Results from these methods reveal the dominance of negative/positive helicity in the northern/southern hemisphere, which is defined as "a rule of helicity sign".

In studying the origin of the twist, Wang et al. (1994) and Leka et al. (1996) used photospheric observation of magnetic fields to probe sub-photospheric fields. They concluded that some active regions were carrying electric currents prior to their emergence. In other words, the twist of the active region magnetic fields was present in a flux tube below the photosphere. There are many models explaining the origin of the twist of magnetic lines in sub-photospheric magnetic flux tubes. Longcope et al. (1996), Moreno-Insertis \& Emonet (1996) and Fan et al. (1998) proposed that twist presented in a flux tube is produced by a solar dynamo before the tube rises. 
Rust \& Kumar (1994) considered that the current helicity is caused by the subphotospheric differential rotation in the convection zone. Longcope \& Klapper (1997) suggested that the twist could be given rise to by the tilt of the magnetic polarity axis, which is caused by the Coriolis force acting on the buoyant, rising flux tube as a $\Omega$-loop in the convection zone. In a recent paper, Longcope et al. (1998) proposed that the twist is imparted to the flux tube through the deformation of the axis of the flux tube, which is caused by turbulent motions with a non-zero kinetic helicity $<\boldsymbol{u} \times(\nabla \times \boldsymbol{u})>$ in the convection zone.

The force-free parameter $\alpha$ and the mean current helicity $<h_{\|}>$calculated in the photosphere carry some information on the twist of magnetic lines in a flux tube rising to the photosphere from the view of observations (Seehafer 1990; Pevtsov et al. 1995; Abramenko et al. 1996; Bao \& Zhang 1998). Positive/negative values of these parameters correspond to the twist of magnetic lines in the right/lefthandedness. We use $<h_{\|}>$as a parameter to describe the twist of an active region magnetic fields in this paper.

It is important for some dynamo and flux tube models to study the origin of the twist of magnetic fields in the active regions. Perhaps, the relationship between the twist of the magnetic field and the tilt angle of an active region could shed light on this problem, as Longcope \& Klapper (1997) and Longcope et al. (1998) have proposed. After considering helicity conservation in a flux tube with zero helicity, helicity modifies both twist and writhe in the tube. However, the writhe will be opposite in sign to the twist (Moffatt \& Ricca 1992) if the Coriolis force produced a twist in originally untwisted flux tubes. Canfield \& Pevtsov (1998) first studied the relationship in sign of both the force-free parameter $\alpha$ and tilt per unit length $\left(-\frac{\theta}{L}\right)$, where $L$ is the separation between sunspots of opposite polarity. Their data show no reliable anticorrelation in sign between twist and writhe (see Fig. 6 of their paper), as one would expect.

However, the mean current helicity $\left\langle h_{\|}>\right.$was measured by Bao \& Zhang (1998) for 422 active regions in the 22 nd cycle. They found that almost $80 \%$ of active regions adhere to the hemispheric helicity rule, being negative in the northern hemisphere and positive in the southern hemisphere. The tilt angle $\varphi$ was measured by Tian et al. (1999) for 203 bipolar regions among the 422 active regions. They found that almost $70 \%$ of bipolar active regions adhere to the Hale-Nickolson Law. What is the relationship in sign between the tilt and the twist using these data? In the present paper, we will use this relationship to investigate where the helicity is produced. Observational techniques and data chosen are described in Sect. 2. Definitions and calculations of tilt angle and current helicity are given in Sect. 3. In Sect. 4, we present the relationship between the sign of the tilt angle of magnetic polarity axis and the sign of the mean current helicity $\left\langle h_{\|}\right\rangle$for 286 bipolar active regions. In Sect. 5, we examine the distribution of active regions with "abnormal chirality". Distribution of 62 X-ray flares larger than M-class is given in Sect. 6. Finally, conclusions and discussions are given in Sect. 7.

\section{Observational techniques and data}

\subsection{Observational technique}

We use the data of vector magnetic fields obtained by the Solar Magnetic Field Telescope, an imaging vector magnetograph installed at Huairou Solar Observing Station of the Beijing Astronomical Observatory, with a field of view of about $5 ! 23 \times 3 ! 63(512 \times 512$ pixels of the $\mathrm{CCD})$. The temporal and spatial resolution of the vector magnetograms depend on the number of video frames that are integrated to make one magnetogram. Each magnetogram used in this paper is the sum of 256 individual frames for both line-of-sight and transverse fields. Temporal resolution is about $5 \mathrm{~min}$ for each vector magnetogram. Each pixel is about $0^{\prime \prime} 6 \times 0 \times 0^{\prime \prime} 4$. After performing a $3 \times 4$ pixels smoothing average, the spatial resolution is $2^{\prime \prime} \times 2^{\prime \prime}$.

The vector magnetic field in the photosphere was obtained on the basis of narrow-band images (filtergrams) of four Stokes parameters $I, V, Q, U$ in FeI 5324.191 $\AA$. The longitudinal magnetic field strength $\left(B_{||}\right)$and transverse magnetic field strength $\left(B_{\perp}\right)$ are given, respectively, by

$B_{\|}=C_{\|} \frac{V}{I}$

$B_{\perp}=C_{\perp}\left(\frac{Q^{2}}{I^{2}}+\frac{U^{2}}{I^{2}}\right)^{\frac{1}{4}}$

where $C_{\|}$and $C_{\perp}$ are the calibration coefficients for lineof-sight and transverse components of the magnetic fields. However, a precise calibration of a vector magnetograph is very complicated and difficult. Both theoretical and empirical methods are used to calibrate the vector magnetograms (Ai et al. 1982; Wang et al. 1996).

The 180-degree azimuthal ambiguity in determining the transverse field direction is an intrinsic defect of the Zeeman effect (Harvey 1969). It may be resolved according to the potential field approximation method (Wang \& Lin 1993; Wang et al. 1994) taking into account the evolution of active regions and the orientation of chromospheric fibrils. After the $3 \times 4$ pixel smoothing average of $V, Q$ and $U$ was made, the noise level was less than $10 \mathrm{G}$ for the line-of-sight field and $100 \mathrm{G}$ for the transverse field over the same integration time.

It should be mentioned that magneto-optical effects (Faraday rotation) is insignificant (Wang et al. 1996) in FeI $5324.191 \AA$. Several tests were made particularly to compare the measured field azimuth for a few sunspots when switching the bandpass from line center to line wing, and differences in the observed azimuth were less than $10^{\circ}$. On the other hand, after Bao et al. (2000) compared the vector magnetograms of an active region, NOAA 5747, from Huairou and Mees Solar Observation, they found a qualitative agreement between them. Then, they estimated that Faraday rotation in the Huairou magnetogram 
contributes about $12^{\circ}$ in the azimuth difference when possible sources of error are taken into account. After considering the role of Faraday rotation in computation of $<h_{\|}>$, Bao et al. (2000) conclude that it does not affect the strength of the hemispheric helicity rule.

\subsection{Data}

In our study, we have chosen 286 active regions which belong to $\beta$ sunspots in the magnetic classification. Most of the active regions are formed by two main bigger spots. Among them, 203 active regions have simpler magnetic configuration (i.e. the magnetic fields in these regions can be simply divided into areas with $\mathrm{N}$ and $\mathrm{S}$ polarities) and 83 are more complicated (i.e. there is some small scale inverse regions in the dominated polarities).

We analyze the vector magnetograms of these active regions obtained from 1988 to 1996 at Huairou Solar Observing Station of the Beijing Astronomy Observatory, and compare them with their images of sunspots in the photosphere and fulldisk magnetograms published in the SGD. It is found that these active region are much better bipolar regions and are isolated from others. The magnetograms always cover the entire active region. All the vector magnetic field data included in this study were carefully chosen and acquired with favorable weather and seeing conditions during the observations. Thus, the noise level could be lowered to $6 \mathrm{G}$ for the line-of-sight field and $60 \mathrm{G}$ for the transverse field in the photosphere. When an active region developed maturely and was located near the central meridian, it is included in the sample. Therefore, most of the active regions are not new emerging and young active regions. We then calculate tilts and current helicity of those active regions using the best magnetograms. The projection effect for high latitude active regions were removed according to the formulae given by Gary \& Hagyard (1990).

\section{Definition of the the tilt angle $\varphi$ and mean current helicity $\left\langle h_{\|}>\right.$}

\subsection{The tilt angle $\varphi$ of magnetic polarity axis}

In calculation of the tilt angle, the magnetic flux-weighted center is determined as the position of each magnetic polarity. This position could be approximately indicated by the magnetic flux weighted center of each magnetic polarity in the line-of-sight as:

$x_{\mathrm{c}}=\frac{\sum x(j, i) B_{\|}(j, i) \mathrm{d} s}{\sum B_{\|}(j, i) \mathrm{d} s}$,

$y_{\mathrm{c}}=\frac{\sum y(j, i) B_{\|}(j, i) \mathrm{d} s}{\sum B_{\|}(j, i) \mathrm{d} s}$

where, $\mathrm{d} s=\mathrm{d} x \mathrm{~d} y$ is the area of each pixel. The tilt angle $(\varphi)$ of an active region is defined as the angle between the line joining opposite polarities and the solar equator. The tangent of the tilt angle $(\tan \varphi)$ is given by $\delta y / \delta x$
(Gary \& Hagyard 1990; Wang \& Sheeley 1989; Howard 1989; Tian et al. 1999), where $\delta y$ and $\delta x$ are the distances between the leading polarity and the following polarity along the $Y$ - and $X$-axis, respectively, in Cartesian coordinates in the heliographic plane.

We determinate the sign of the tilt angle of the magnetic polarity axis in an active region magnetic field such that: in the northern hemisphere, if the $S$ polarity is closer to the solar equator, the tilt of the active region is positive $(0<\varphi<180$ degree $)$, otherwise the tilt is negative $(0>\varphi>-180$ degree $)$; in the southern hemisphere, the tilt is negative $(0>\varphi>-180$ degree $)$ when the $N$ polarity is closer to the equator, and otherwise the tilt is positive $(0<\varphi<180$ degree $)$. Note that in the 22 nd solar cycle, the leading spot should be $S / N$-polarity in the northern/southern hemisphere. Thus, active regions with a tilt angle $0<\varphi<90$ degree in the northern hemisphere, or $-90<\varphi<0$ degree in the southern hemisphere, all adhere to Joy's Law. Figure 1 shows the sign convention of the tilt angles $(\varphi)$ in the 22 nd solar cycle.
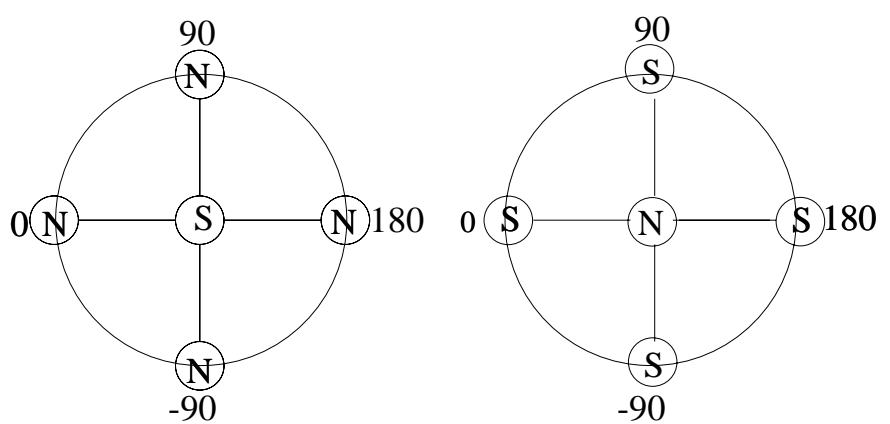

In the Northern Hemisphere In the Southern Hemisphere

Fig. 1. Convention for the sign of the tilt angle of the magnetic polarity axis. Active regions with a tilt angle $0<\varphi<90$ degree in the northern hemisphere, or $-90<\varphi<0$ degree in the northern hemisphere, follow Joy's law.

In order to obtain the flux-weighted center closer to the center of the sunspot and then the tilt of active regions, a magnetic field of $160 \mathrm{G}$ was selected as a criterion of the minimum of magnetic strength, so that the effect of some other weak magnetic fields around main polarities would be minimized. It is useful for us to calculate the flux-weighted center and tilt angle of magnetic polarity axis by this method (Tian et al. 1999).

\subsection{Mean current helicity $\left\langle h_{\|}>\right.$}

Current helicity plays an important role in the study of twisted magnetic fields in the solar atmosphere. Its density can be split into two parts,

$$
\begin{aligned}
h_{\mathrm{c}} & =\boldsymbol{B} \cdot(\nabla \times \boldsymbol{B}) \\
& =\boldsymbol{B}_{\|} \cdot(\nabla \times \boldsymbol{B})_{\|}+\boldsymbol{B}_{\perp} \cdot(\nabla \times \boldsymbol{B})_{\perp} \\
& =h_{\|}+h_{\perp},
\end{aligned}
$$


$h_{\|}$is the parallel component in the direction of the line of sight which can be inferred from photospheric vector magnetograms. $h_{\perp}$ is the transverse component which is hard to compute because we have magnetic measurement only at a single height in the solar atmosphere.

In the approximation of the force-free magnetic field: $\nabla \times \boldsymbol{B}=\alpha_{\mathrm{f}} \boldsymbol{B}$, there is,

$\alpha_{\mathrm{f}}=\frac{(\nabla \times \boldsymbol{B})_{\|}}{\boldsymbol{B}_{\|}}=\frac{\boldsymbol{B}_{\|} \cdot(\nabla \times \boldsymbol{B})_{\|}}{\boldsymbol{B}_{\|}^{2}}$.

Thus, the density of current helicity is:

$$
\begin{aligned}
h_{\mathrm{c}} & =\boldsymbol{B} \cdot(\nabla \times \boldsymbol{B})=\alpha_{\mathrm{f}} B^{2} \\
& =\frac{\boldsymbol{B}^{2}}{\boldsymbol{B}_{\|}^{2}}\left(\boldsymbol{B}_{\|} \cdot(\nabla \times \boldsymbol{B})_{\|}\right) .
\end{aligned}
$$

Here, we define

$$
<h_{\|}>=<\boldsymbol{B}_{\|} \cdot(\nabla \times \boldsymbol{B})_{\|}>\equiv \mu_{0}<\boldsymbol{B}_{\|} \cdot \boldsymbol{J}_{\|}>,
$$

as mean of $h_{\|}$distribution over an active region, where $\mu_{0}=4 \pi \times 10^{-3} \mathrm{GmA}^{-1}$, and the vertical current density $J_{\|}$can be expressed by

$J_{\|}=\frac{1}{\mu_{0}}\left(\frac{\partial B_{y}}{\partial x}-\frac{\partial B_{x}}{\partial y}\right)$

(Wang et al. 1994).

In order to minimize the effect of observational error, the current helicity $h_{\|}$is calculated only in pixels as $B_{\|}>20 \mathrm{G}, B_{\perp}>100 \mathrm{G}$ and current density $J_{\|}>$ $0.001 \mathrm{Am}^{-2}$, with the noise level of $J_{\|}(j, i)$. Eventually, Bao et al. (1998) determined an average of the current helicity for each active region. In this case, the uncertainty of magnetic fields affects $\left\langle h_{\|}\right\rangle$very little. We evaluated the error in $2 \sigma$ in Figs. 2 and 3. However, although an active region develops over days, the sign of $\left\langle h_{\|}>\right.$and the sign of the tilt angle of the active region do not change.

\section{Relationship between the sign of the tilt angle and the sign of the mean current helicity $<\boldsymbol{h}_{\|}>$}

We have calculated the tilt angle $\varphi$ in the paper by Tian et al. (1999) and current helicity $\left\langle h_{\|}>\right.$in the paper by Bao \& Zhang (1998) for the 286 active regions. Thus, we can investigate the relationship between the sign of the tilt angle of the magnetic polarity axis and the sign of the current helicity $\left\langle h_{\|}\right\rangle$. Figure 2 illustrates this relationship for 203 bipolar active regions with relatively simple configurations of magnetic fields.

Figure 3 shows such relationship for the other 83 bipolar active regions with relatively more complicated magnetic fields than the 203 active regions. We find that in both kinds of bipolar active regions, about $60 \%$ of the regions have a positive/negative tilt angle and a negative/positive current helicity in the northern/southern hemisphere. In other words, there is a negative correlation between the sign of the tilt angle and the sign of the
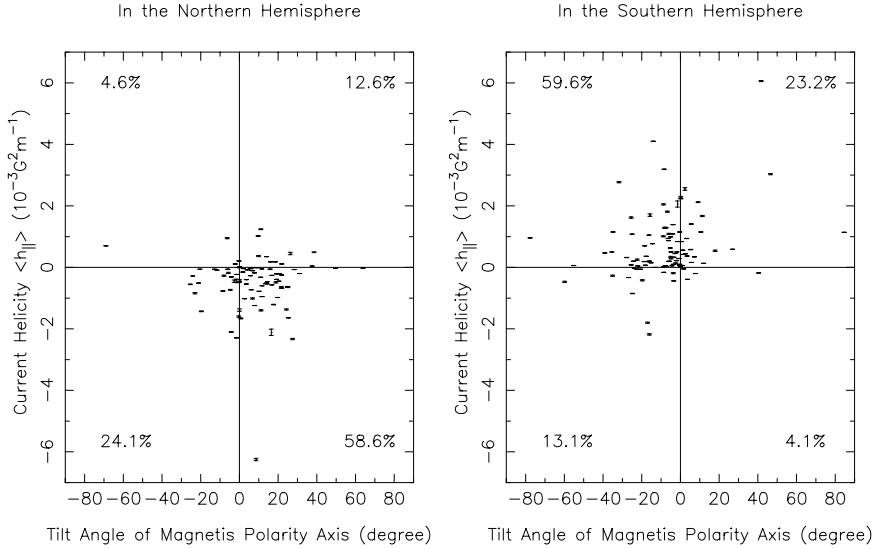

Fig. 2. Relationship between the tilt angle $\varphi$ and the mean current helicity $\left\langle h_{\|}\right\rangle=\left\langle\boldsymbol{B}_{\|} \cdot(\nabla \times \boldsymbol{B})_{\|}\right\rangle$for 203 bipolar active regions. In these active regions, the magnetic configuration is simple. Each point denotes an active region. The length of error bars is determined $2 \sigma$.
In the Northern Hemisphere

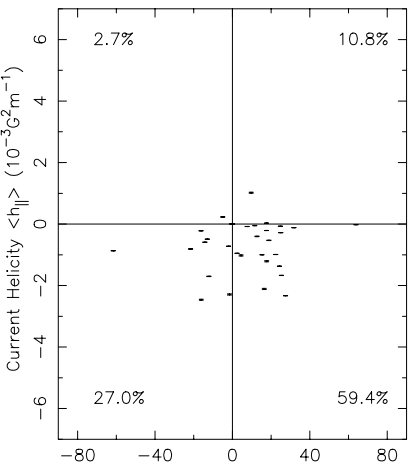

Tilt Angle of Magnetic Polarity Axis (degree)
In the Southern Hemisphere

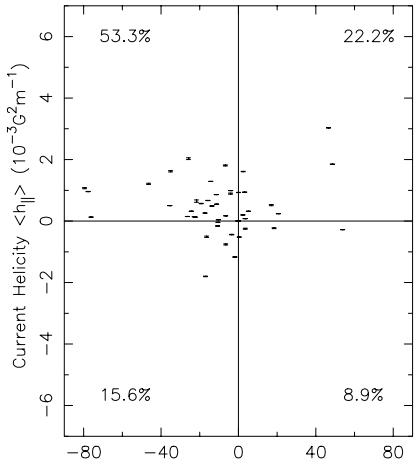

Tilt Angle of Magnetic Polarity Axis (degree)
Fig. 3. The relationship between the tilt angles $\varphi$ and the mean current helicity $<(\nabla \times \mathbf{B})_{\|} \cdot \boldsymbol{B}_{\|}>$for 83 bipolar active regions. In these active regions, magnetic configurations are more complicated. The sign convention of the tilt angle and the current helicity is same as in Fig. 2. The length of error bars is determined $2 \sigma$. Each point denotes an active region.

mean twist. These active regions adhere not only to Joy's Law (Hale et al. 1919), but also to the hemispheric helicity rule (Bao \& Zhang 1998; Pevtsov et al. 1995). Thus, we call these active regions "normal active regions".

If we consider an active region as an $\Omega$-flux tube rising through the convection zone (Babcock 1961) to the photosphere, the tube acquires writhe through the Coriolis force on internal flow (Fan et al. 1993). The tilt of active regions with respect to the equator is an observable manifestation of such writhe, at photospheric level. A positive/negative tilt angle corresponds to the right/left-handed writhe of the flux tube in the northern/southern hemisphere on the basis of our sign convention for the tilt angle in Fig. 1. On the whole, a negative/positive mean current helicity $<h_{\|}>=<(\nabla \times \boldsymbol{B})_{\|} \cdot \boldsymbol{B}_{\|}>$approximately reflects a left/right-handed mean twist of magnetic lines. Thus, these normal active regions are in a stable condition because of the minimization of total helicity. 


\section{Distribution of active regions with different chirality}

Figures 2 and 3 show that most of the active regions in the northern/southern hemisphere have a positive/negative tilt angle and a negative/positive mean twist $<h_{\|}>$of the magnetic field lines. These active regions are regarded as "normal active regions" with "normal chirality" due to adhering to both Joy's Law and the hemispheric helicity rule. While in some other active regions, as in Figs. 2 and 3 , we see the sign of the tilt angle is the same as the sign of the mean twist $<h_{\|}>$. These active regions disobey either Joy's Law or the hemispheric helicity rule. We regard these regions as "abnormal active regions" with "abnormal chirality". Our results show that in both the northern and southern hemisphere, about $60 \%$ of the active regions are of "normal chirality", and about one-third of the active regions are of "abnormal chirality".

In our data, there are a third kind of active region which has a negative/positive tilt angle with a positive/negative twist in the northern/southern hemisphere, i.e. adhering to neither Joy's Law nor the hemispheric helicity rule. They do not belong to either of the two groups defined above and are a tiny fraction of the active regions (less than 5\%).
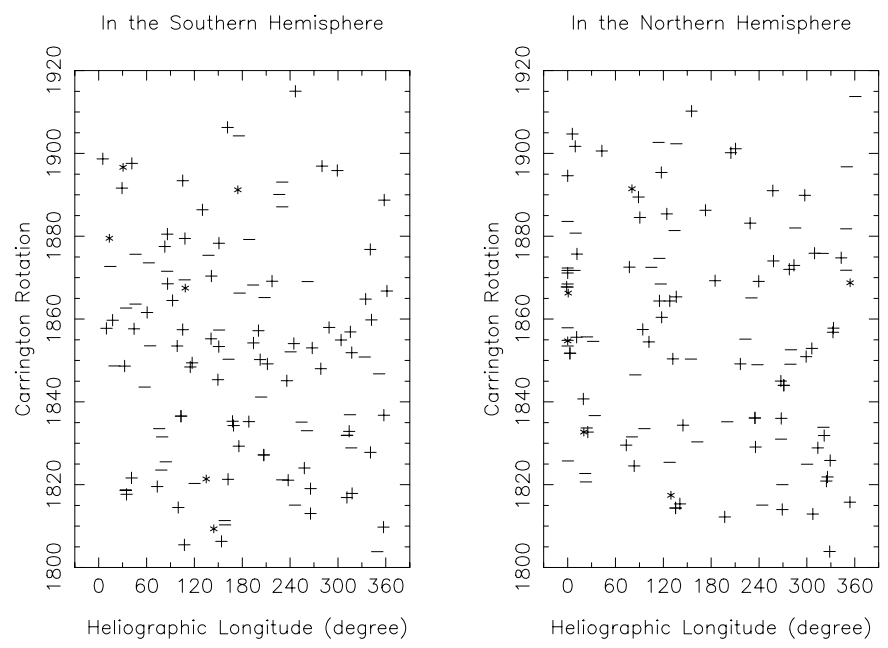

Fig. 4. Longitudinal distribution of the active regions with Carrington rotation. The sign "+" denotes active regions with a "normal chirality", "-" denotes active regions with an "abnormal chirality", and "*" denotes active regions with neither a "normal chirality" nor an "abnormal chirality" (see text). Each point indicates one active region.

In Fig. 4 we give the distribution of the studied active regions with Carrington rotation. In the figure, the sign "+" denotes active regions with "normal chirality", i.e., active regions with a positive/negative tilt angle $\varphi$ and a negative/positive twist $<h_{\|}>$of magnetic lines in the northern/southern hemisphere; "_" denotes active regions with an "abnormal chirality", i.e., active regions in which the tilt angle $\varphi$ and the mean twist $\left\langle h_{\|}\right\rangle$ have the same sign. The third kind of active region with a negative/positive tilt angle $\varphi$ and a positive/negative twist $\left\langle h_{\|}>\right.$in the northern/southern hemisphere, which have neither a "normal chirality" nor an "abnormal chirality", are denoted by "*". Figure 4 show that some areas keep the same sign for many rotations, implying that the helicity of the magnetic fields has been generated at great depths. This result is in agreement with Canfield \& Pevtsov (1998). We especially find that those active regions with "abnormal chirality", denoted by "." sign, tend to be located at some special heliospheric longitudes, such as $0-30^{\circ}, 120^{\circ}, 240^{\circ}-270^{\circ}$ and $330^{\circ}-360^{\circ}$ in the northern hemisphere and $30^{\circ}-90^{\circ}, 180^{\circ}-270^{\circ}$ in the southern hemisphere.

\section{Distribution of 62 major X-ray flares in the 22nd activity cycle}

Bai (1988) examined the distribution of some active regions which produced eruptive flare during the 19th, 20th and 21st solar cycles. He found that these flares were located at some special longitudes. We also examine the longitudinal distribution of active regions that produced major flares with proton events in the 22nd solar cycle. Figure 5 shows the distribution of these active regions with major flares in the heliosphere. It is found that these active regions with major flares also tend to be located at some special longitudes.

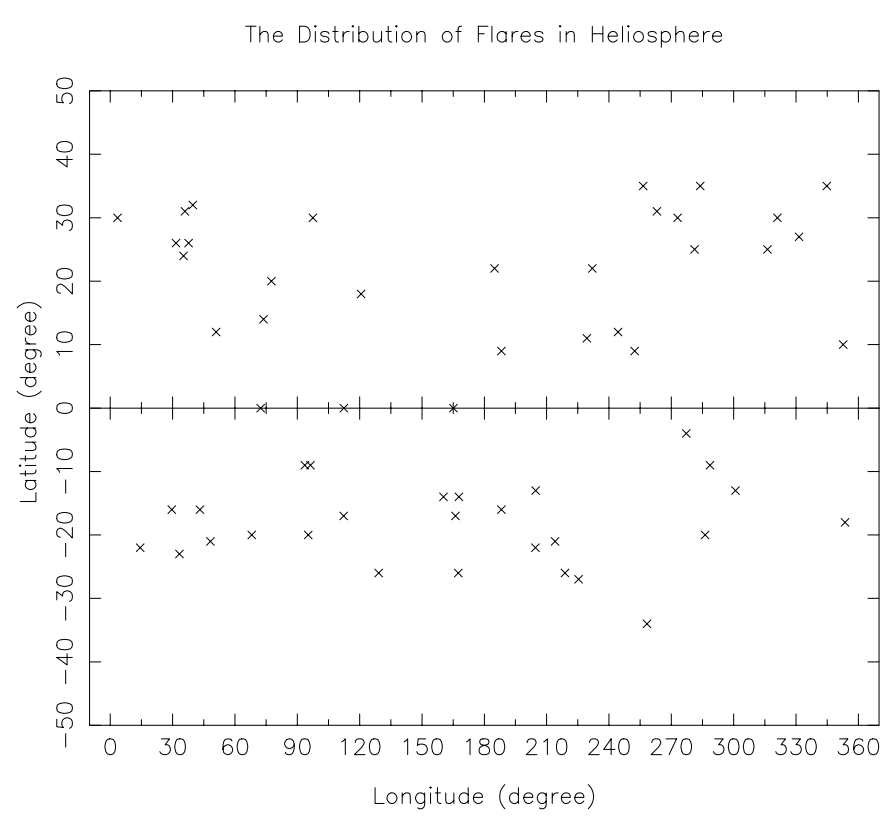

Fig. 5. Distribution of $62>$ M-class X-ray flares with proton event in the 22nd activity cycle. Each point denotes an active region.

Figure $6 \mathrm{a}$ is the histogram used to show the number of the major flares in $30^{\circ}$ longitudinal bins. We note that most of the major flares tend to occur in the longitudinal bins $15^{\circ}-45^{\circ}, 225^{\circ}-285^{\circ}$ and $315^{\circ}-345^{\circ}$ in the northern hemisphere and bins $15^{\circ}-105^{\circ}, 165^{\circ}-225^{\circ}$ and $285^{\circ}$ in the southern hemisphere. Figure $6 \mathrm{~b}$ is the histogram used 
to show the number of active regions in the $30^{\circ}$ longitudinal bins. Solid line denotes the distribution of the active regions with "abnormal chirality", shown as "-" sign in Fig. 4. The dotted line denotes the distribution of the active regions with "normal chirality", shown as "+" in Fig. 4. We notice that in the longitudinal bins with more major flares, there are more active regions with "abnormal chirality" (shown as shadowed regions A to F). These active regions with "normal chirality" have their longitudinal bins preferred.
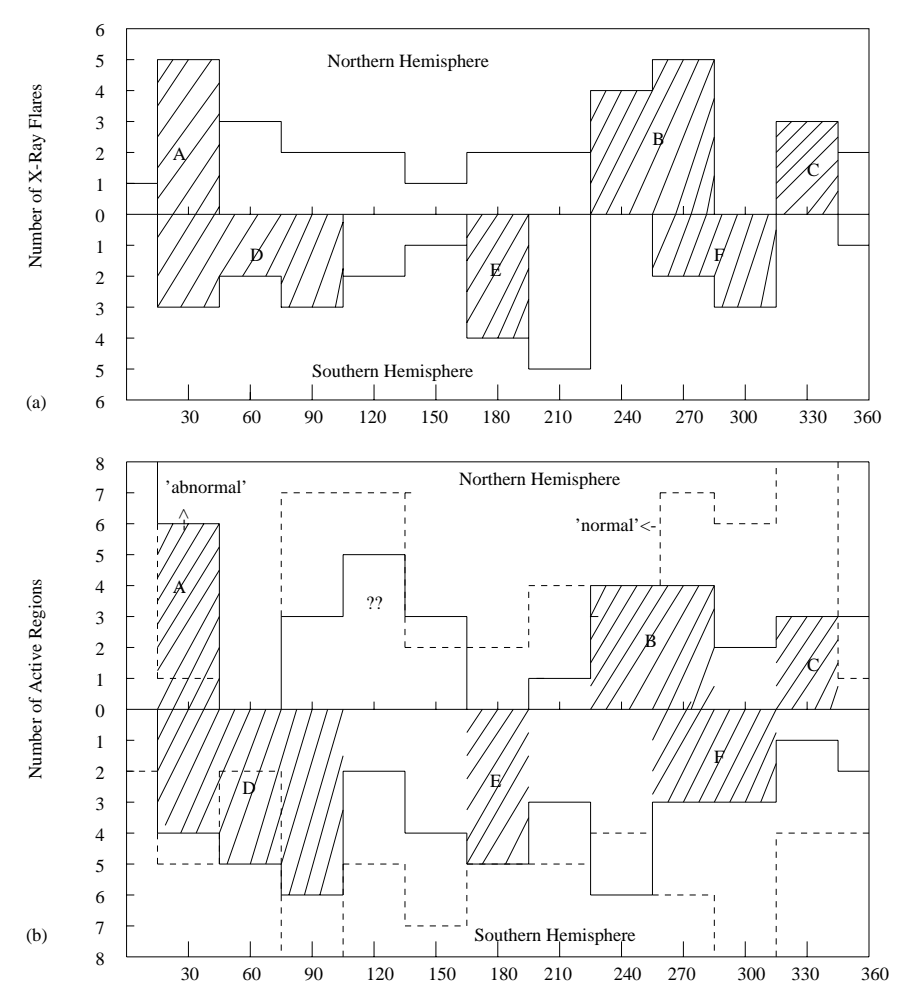

Fig. 6. a) Histogram of distribution of the $62>$ M-class Xray flares in the 22 nd cycle in the $30^{\circ}$ longitudinal bins. b) Histogram of distribution of the active regions. Solid line denotes the active regions with "abnormal chirality", shown as "-" sign in Fig. 4. Dotted line denotes the active regions with "normal chirality", showed as "+" sign in the Fig. 4.

\section{Conclusions and discussions}

We note the following results from this study:

(1) About $60 \%$ of studied bipolar active regions are normal active regions with "normal chirality". It is inferred that the tilt angle of the magnetic polarity axis and the mean current helicity $\left\langle h_{\|}\right\rangle$have opposite signs. In other words, there is a negative correlation between the sign of the tilt angle and the sign of the mean twist.

(2) About one-third of bipolar active regions are abnormal active regions with the "abnormal chirality". In these active regions, the tilt angles and the current helicity have the same sign. These active regions tend to be located at particular longitudes in the heliosphere.
(3) Most of the $62 \mathrm{X}$-ray flares larger than M-class in the 22nd cycle also tend to occur in particular longitudinal bins, where active regions with "abnormal chirality" appear frequently.

We know that total helicity $H$ of a flux tube can be separated into the twist helicity $T_{\mathrm{w}}$ of magnetic lines in the tube and the writhe helicity $W_{\mathrm{r}}$ of the tube (Moffatt \& Ricca 1992). Therefore, an active region with a "normal chirality" (i.e., a left/right handed twist accompanied by a right/left handed writhe) is likely to be in a lower energy state due to a negative/positive twist helicity and a positive/negative writhe helicity. This is a relatively stable tube because the total helicity is minimized. An active region with an "abnormal chirality" (i.e., a left or right handed twist and a same handed writhe) is likely to be in a higher energy state due to the same sign of twist and writhe helicity. There is much more chance of magnetic reconnection in this kind of active region. Therefore, we propose that the "abnormal chirality" of active regions is a possible source of instability able to produce some eruptive phenomena as seen in the Fig. 6. Fisher et al. (2000) predict that the twist and writhe in $\delta$-spot active regions should have the same sign because of the kink hypothesis for $\delta$-spot active regions. The active region with an "abnormal chirality" may represent these kinked regions. However, the relevance between the distribution of the flares and that of the active regions with "abnormal chirality" is not high (shown in the Fig. 6). We believe that it is probably because the number of cases is relatively low for statistical significance.

From Figs. 2 and 3, we find that there is a roughly fixed percentage in each quarter. We believe this is not a coincidence and that there must be a physical reason. Canfield \& Pevtsov (1998) use a different twist indicator, the force-free parameter $\alpha$, to investigate the relationship in sign between the tilt and the twist of magnetic fields. They obtain important and some what different results from ours. What is the reason? Is it because they use different twist parameters of magnetic fields? Is there another physical meaning? Figure 4 shows us that some longitudes keep the same or opposite sign between the tilt and twist for many rotations, that tells us that the twist of magnetic fields is produced at great depths, not on the surface. What is real origin of the twist of the magnetic fields?

This study could give us some information on the chirality of the magnetic fields in the sub-photospheric convection zone. We will further investigate the origin and development of the magnetic field twist and the tilt of active regions, which is an important respect of some dynamic models of sub-photospheric flux tubes and the dynamo theory as well. A following paper will discuss this question.

Acknowledgements. The authors are grateful to Drs. T. J. Wang, Y. H. Yan, and Y. Y. Deng for useful discussions, valuable comments and suggestions. They specially acknowlede Dr. Jiong Qiu who helped us to improve this paper. The authors are grateful to the referee for his/her helpful suggestions. 
This research is supported by NSFC Grant Nos. 19791090 and 10073013.

\section{References}

Abramenko, V. I., Wang, T. J., \& Yurchishin, V. B. 1996, Solar Phys., 168, 75

Ai, G. X., Li, W., \& Zhang, H. Q. 1982, Chinese Astron. \& Astrophys., 6, 129

Babcock, H. W. 1961, ApJ, 133, 572

Bai, T. 1988, ApJ, 328, 860

Bao, S. D., \& Zhang, H. Q. 1998, ApJ, 496, L43

Bao, S. D., Pevtsov, A. A., Wang, T. J., \& Zhang, H. Q. 2000, Solar Phys., 195, 75

Bieber, J. W., Evenson, P. A., \& Matthaeus, W. H. 1987, ApJ, 315,700

Burlaga, L. F. 1988, Geophys. J. Res., 93(A7), 7217

Canfield, R., \& Pevtsov, A. 1998, Synoptic Solar Physics, ASP Conf. Ser. 140, ed. K. Balasubramaniam, J. Harvey, \& D. Rabin, 131

Fan, Y., Fisher, G. H., \& DeLuca, E. E. 1993, ApJ, 405, 390

Fan, Y., Zweibel, E. G., \& Lantz, S. R. 1998, ApJ, 493, 480

Fisher, G., Fan, Y., Longcope, D., Linton, M., \& Pevtsov, A. 2000, Solar Phys., 192, 119

Gary, G. A., \& Hagyard, M. J. 1990, Solar Phys., 126, 21

Hale, G. E., Ellerman, F., Nicholson, S. B., \& Joy, A. H. 1919, ApJ, 49, 153

Hagyard, M., \& Pevtsov, A. 1999, Solar Phys., 189, 25

Harvey, J. W. 1969, Ph.D. Thesis, Univ. of Colorado

Howard, R. F. 1989, Solar Phys., 123, 271

Howard, R. F. 1991, Solar Phys., 132, 49

Leka, K. D. , Canfield, R. C., McClymont, A. N., \& Van DrielGesztelyi, L. 1996, ApJ, 426, 547
Longcope, D. W., Fisher, G. H., \& Arendt, S. 1996, ApJ, 464, 999

Longcope, D. W., \& Klapper, I. 1997, ApJ, 488, 443

Longcope, D. W., Fisher, G. H., \& Pevtsov, A. A. 1998, ApJ, 507,417

Martin, S. F., Billamoria, R., \& Tracadas, P. W. 1994, in Solar Surface Magnetism, ed. R. J. Rutten, \& C. J. Schrijver (Dordrecht: Kluwer), 303

Moffatt, H. K., \& Ricca, R. L. 1992, Proc. R. Soc. Lond. A, 439, 411

Moreno-Insertis, F., \& Emonet, T. 1996, ApJ, 472, L53

Pevtsov, A. A., Canfield, R. C., \& Metcalf, T. R. 1995, ApJ, 440, L109

Rust, D. M., \& Kumar, A. 1994, Solar Phys., 155, 69

Rust, D. M., \& Kumar, A. 1996, ApJ, 464, L199

Schmidt, H. U. 1968, in IAU Symp., 35, Structure and Development of Solar Active Regions, ed. K. O. Kiepeuheuer, 95

Seehafer, N. 1990, Solar Phys., 125, 219

Tian, L., Zhang, H., Tong, Y., \& Jing, H. 1999, Solar Phys., 189,305

Wang, H. N., \& Lin, Y. 1993, Chin. Astron. Astrophys., 17, 423

Wang, J. X., Shi, Z. X., Wang, H. N., \& Lü, Y. P. 1996, ApJ, 456,861

Wang, T. J., Xu, A. A., \& Zhang, H. Q. 1994, Solar Phys., 155, 99

Wang, T. J., Ai, G. X., Deng, Y. Y. 1996, Publ. Beijing Astron. Obs., 28, 31

Wang, Y.- M., \& Sheeley, N. R. 1989, Solar Phys., 124, 81

Wang, Y. M., \& Sheeley, N. R. 1991, ApJ, 375, 761 